\title{
JORDAN HOMOMORPHISMS ONTO PRIME RINGS
}

\author{
BY \\ M. F. SMILEY
}

1. Introduction. In a recent paper $\left(^{1}\right)$ I. N. Herstein proved that a Jordan homomorphism onto a prime ring of characteristic different from two and three is either a homomorphism or an anti-homomorphism. The purpose of this note is to give a brief proof of this result and at the same time remove the requirement that the characteristic be different from three. We rely on a lemma, proved in $\$ 3$, to the effect that a prime ring in which the square of every commutator is zero must be commutative and hence free from nonzero divisors of zero. We use a number of the preliminary results of Herstein, and hence we adopt notations which conform with his. However, since it is almost as easy to prove these preliminary results as to state them, we include their proofs and make our presentation completely self-contained.

It is with pleasure that the author acknowledges that certain conversations with his wife, Dorothy M. Smiley, aided materially in the derivation of the results given in this note.

2. Herstein's theorem. In an associative ring $R$, we write $a \cdot b=a b+b a$ and $[a, b]=a b-b a$ for the Jordan product and the commutator of $a, b \in R$. A mapping $a \rightarrow a^{\prime}$ of a ring $R$ into a ring $R^{\prime}$ is called a Jordan homomorphism $[\mathrm{H}]$ in case $(a+b)^{\prime}=a^{\prime}+b^{\prime}$ and $(a \cdot b)^{\prime}=a^{\prime} \cdot b^{\prime}$ for every $a, b \in R$. We assume that $2 a^{\prime}=0$ for $a^{\prime} \in R^{\prime}$ implies that $a^{\prime}=0$. Then $(a \cdot a)^{\prime}=a^{\prime} \cdot a^{\prime}$ yields $\left(a^{2}\right)^{\prime}$ $=\left(a^{\prime}\right)^{2}$ for every $a \in R$; while $((a \cdot b) \cdot a)^{\prime}=\left(a^{\prime} \cdot b^{\prime}\right) \cdot a^{\prime}$ then gives $\left(2 a b a+b \cdot a^{2}\right)^{\prime}$ $=2 a^{\prime} b^{\prime} a^{\prime}+b^{\prime} \cdot\left(a^{\prime}\right)^{2}$, and hence $(a b a)^{\prime}=a^{\prime} b^{\prime} a^{\prime}$ for every $a, b \in R$. Linearizing, we have $(a b c+c b a)^{\prime}=a^{\prime} b^{\prime} c^{\prime}+c^{\prime} b^{\prime} a^{\prime}$ for every $a, b, c \in R$. We adopt the abbreviations $a^{b}=(a b)^{\prime}-a^{\prime} b^{\prime}, a_{b}=(a b)^{\prime}-b^{\prime} a^{\prime}$ of Herstein [H] and note that (0) $b^{a}=-a^{b}, b_{a}=-a_{b}$,

$$
\left[a^{\prime}, b^{\prime}\right]=a_{b}-a^{b},
$$

$$
[a, b]^{\prime}=2(a b)^{\prime}-(a \cdot b)^{\prime}=a_{b}+a^{b}
$$

for every $a, b \in R$, Now compute

$$
a^{b} a_{b}=\left((a b)^{\prime}-a^{\prime} b^{\prime}\right)\left((a b)^{\prime}-b^{\prime} a^{\prime}\right)=\left((a b)^{2}+a b^{2} a-(a b) b a-a b(a b)\right)^{\prime}=0
$$

for every $a, b \in R$. By right-left duality, using (0), we also have $a_{b} a^{b}=0$ for

Presented to the Society, November 23, 1956; received by the editors May 22, 1956.

(1) I. N. Herstein, Jordan homomorphisms, Trans. Amer. Math. Soc. vol. 81 (1956) pp. 331-341. We shall refer to this paper as H. Herstein gives a review of the history of Jordan homomorphisms, but he fails to mention that the concept of "prime ring" is due to Neal $\mathrm{H}$. McCoy, Prime ideals in general rings, Amer. J. Math. vol. 71 (1949) pp. 823-833. We shall also need to refer to the paper of N. Jacobson and C. E. Rickart, Jordan homomorphisms of rings, Trans. Amer. Math. Soc. vol. 69 (1950) pp. 479-502. We shall refer to this paper as JR. 
every $a, b \in R$. If $a, b, r \in R$, then

$$
r^{\prime} a^{b}+a_{b} r^{\prime}=r^{\prime} \cdot(a b)^{\prime}-r^{\prime} a^{\prime} b^{\prime}-b^{\prime} a^{\prime} r^{\prime}=(r \cdot(a b)-r a b-b a r)^{\prime}=([a, b] r)^{\prime} .
$$

Thus we obtain

$$
r^{\prime} a^{b}+a_{b} r^{\prime}=([a, b] r)^{\prime}
$$

for every $a, b, r \in R$. All of the results we have derived so far may be found in $[\mathrm{H}]$ and most of them also in [JR]. No further references to previous work will be required. We assume for the remainder of this section that $R^{\prime}$ is a prime ring and that $a \rightarrow a^{\prime}$ is a Jordan homomorphism of $R$ onto $R^{\prime}$.

The right-left dual of (3) is

$$
a^{b} \boldsymbol{r}^{\prime}+\boldsymbol{r}^{\prime} a_{b}=(\boldsymbol{r}[a, b])^{\prime}
$$

for every $a, b, r \in R$. Since $a^{b} a_{b}=0$, from (3) we obtain

$$
a^{b} r^{\prime} a^{b}=a^{b}([a, b] r)^{\prime}
$$

for every $a, b, r \in R$. Also $a^{b} a_{b}=0$ and (3) give

$$
a_{b} r^{\prime} a_{b}=([a, b] r)^{\prime} a_{b}
$$

for every $a, b, r \in R$. We follow Herstein and replace $r$ by $[a, b] r$ in (4), use (5), (6), $(x y x)^{\prime}=x^{\prime} y^{\prime} x^{\prime}$, and (2) to obtain $a^{b} r^{\prime} a^{b}+a_{b} r^{\prime} a_{b}=\left(a_{b}+a^{b}\right) r^{\prime}\left(a_{b}+a^{b}\right)$, or

$$
a_{b} r^{\prime} a^{b}+a^{b} r^{\prime} a_{b}=0
$$

for every $a, b, r \in R$. This is the key identity, as we shall see.

Let us assume that $\left(c^{d}\right)^{2} \neq 0$ for some $c, d \in R$. Then (7) and $c^{d} c_{d}=0$ give $\left(c^{d}\right)^{2} r^{\prime} c_{d}=0$ for all $r^{\prime} \in R^{\prime}$ and hence $c_{d}=0$ because $R^{\prime}$ is a prime ring. Partial linearizations of (7) and of $a^{b} a_{b}=0$ are

$$
\begin{aligned}
a_{b} r^{\prime} a^{x}+a_{x} r^{\prime} a^{b}+a^{b} r^{\prime} a_{x}+a^{x} r^{\prime} a_{b} & =0, \\
a^{b} a_{x}+a^{x} a_{b} & =0,
\end{aligned}
$$

for every $a, b, x, r \in R$. Setting $a=c$ and $b=d$ in (8) and (9) yields $c_{x} r^{\prime} c^{d}$ $+c^{d} r^{\prime} c_{x}=0$ and $c^{d} c_{x}=0$ for every $x, r \in R$. But then $\left(c^{d}\right)^{2} r^{\prime} c_{x}=0$, and $c_{x}=0=x_{c}$ for every $x \in R$ because $R^{\prime}$ is a prime ring. Since $\left(c^{d}\right)^{2}=\left(d^{c}\right)^{2} \neq 0$, we also have $d_{x}=x_{d}=0$ for every $x \in R$. A complete linearization of (7) gives

$$
a^{b} \boldsymbol{r}^{\prime} y_{x}+a^{x} \boldsymbol{r}^{\prime} y_{b}+y^{b} \boldsymbol{r}^{\prime} a_{x}+y^{x} \boldsymbol{r}^{\prime} a_{b}+a_{b} \boldsymbol{r}^{\prime} y^{x}+a_{x} \boldsymbol{r}^{\prime} y^{b}+y_{b} \boldsymbol{r}^{\prime} a^{x}+y_{x} \boldsymbol{r}^{\prime} a^{b}=0
$$

for every $a, b, r, x, y \in R$. Take $a=c$ and $b=d$ in this identity to obtain

$$
c^{d} \boldsymbol{r}^{\prime} y_{x}+y_{x} \boldsymbol{r}^{\prime} c^{d}=0
$$

for every $x, y \in R$. A complete linearization of $a^{b} a_{b}=0$ gives

$$
a^{b} y_{x}+y^{b} a_{x}+a^{x} y_{b}+y^{x} a_{b}=0
$$


for every $a, b, x, y \in R$. Setting $a=c$ and $b=d$ gives $c^{d} y_{x}=0$ for every $x, y \in R$. This result, combined with (10), yields $\left(c^{d}\right)^{2} r^{\prime} y_{x}=0$ and consequently $y_{x}=0$ for every $x, y \in R$ because $R^{\prime}$ is a prime ring.

In an analogous way we may see that $\left(c_{d}\right)^{2} \neq 0$ for some $c, d \in R$ implies that $y^{x}=0$ for every $x, y \in R$. Thus one of the following three alternatives holds.

$$
\begin{aligned}
& \text { We have } y^{x}=0 \text { for every } x, y \in R . \\
& \text { We have } x_{y}=0 \text { for every } x, y \in R .
\end{aligned}
$$

$$
\text { We have }\left(x_{y}\right)^{2}=\left(x^{y}\right)^{2}=0 \text { for every } x, y \in R \text {. }
$$

If the alternative (13) prevails, then (1), together with $a^{b} a_{b}=a_{b} a^{b}=0$ for every $a, b \in R$, shows that

$$
\left[a^{\prime}, b^{\prime}\right]^{2}=0 \text { for every } a^{\prime}, b^{\prime} \in R^{\prime} .
$$

By the lemma of the following section, when (14) holds in a prime ring $R^{\prime}$, then $R^{\prime}$ is commutative and is, therefore, free of nonzero divisors of zero. This completes the proof of Herstein's theorem, including the case of characteristic three. We state this result as a theorem for the sake of completeness.

TheOREM. Let $a \rightarrow a^{\prime}$ be a mapping of a ring $R$ onto a prime ring $R^{\prime}$ and assume that (1) $2 a^{\prime}=0$ for $a^{\prime} \in R^{\prime}$ implies that $a^{\prime}=0$, and that (2) $(a b+b a)^{\prime}$ $=a^{\prime} b^{\prime}+b^{\prime} a^{\prime}$ and $(a+b)^{\prime}=a^{\prime}+b^{\prime}$ for every $a, b \in R$. Then either $(a b)^{\prime}=a^{\prime} b^{\prime}$ for every $a, b \in R$ or $(a b)^{\prime}=b^{\prime} a^{\prime}$ for every $a, b \in R$.

3. A lemma on prime rings. We isolate in this section a result which may have some interest beyond its application to the study of Jordan homomorphisms onto prime rings.

Leмma. Let $P$ be a prime ring such that

$$
[x, y]^{2}=0
$$

for every $x, y \in P$. Then $P$ is commutative (and is, therefore, free of nonzero divisors of zero).

Proof. Linearize (15) a bit to obtain

$$
[x, y][x, z]+[x, z][x, y]=0
$$

for every $x, y, z \in P$, i.e., two commutators with an element in common anti-commute. Then, by (16) and (15), we have, for $x, y, z, t \in P$,

$$
[x, y][x, t[z, y]][x, y]=0 \text {. }
$$

Now we apply the semi-Jacobi identity: $[a, b c]=b[a, c]+[a, b] c$ to obtain

$$
[x, y] t[x,[z, y]][x, y]+[x, y][x, t][z, y][x, y]=0
$$

for every $x, y, z, t \in P$. The second term of this equation vanishes by (16) and 
(15). Since $P$ is a prime ring we have $[x, y]=0$ for every $x, y \in P$ and our conclusion or we obtain for $x, y, z \in P$,

$$
[x,[z, y]][x, y]=0 .
$$

Interchange $x$ and $y$ to get

$$
[y,[x, z]][x, y]=0
$$

for every $x, y, z \in P$. The Jacobi identity then gives

$$
[z,[x, y]][x, y]=0,
$$

which, in view of (15) yields

$$
[x, y] z[x, y]=0
$$

for every $x, y, z \in P$. Since $P$ is a prime ring, $[x, y]=0$ for every $x, y \in P, P$ is commutative, and the proof of the lemma is complete.

Added in proof (February 16, 1957). We have used the requirement (1) of the theorem only in our derivation of $\left(1^{*}\right)\left(a^{2}\right)^{\prime}=\left(a^{\prime}\right)^{2}$ and $(a b a)^{\prime}=a^{\prime} b^{\prime} a^{\prime}$ for every $a, b \in R$. Thus $\left(1^{*}\right)$ may replace $(1)$ in the theorem. The requirement $\left(1^{*}\right)$ is used in JR to define Jordan homomorphisms effectively for arbitrary rings.

State University OF Iowa, Iowa City, Iowa. 